\title{
Novedades para la flora vascular del Espacio Natural Sierra de las Nieves (Málaga, España)
}

Federico Casimiro-Soriguer Solanas, Andrés V. Pérez Latorre \& Baltasar Cabezudo

Área de Botánica (Departamento de Biología Vegetal). Facultad de Ciencias. Universidad de Málaga. Málaga

\section{Correspondencia}

F. Casimiro-Soriguer Solanas

e-mail: fedeque@hotmail.com

Recibido: 14 mayo 2019

Aceptado: 17 septiembre 2019

Publicado on-line: 24 septiembre 2019

Editado por: E. Bañares

\section{New data for the vascular flora of Natural area Sierra de las Nieves}

Palabras clave: Flora vascular, Andalucía, Espacios Naturales Protegidos.

Key words: Vascular Flora, Andalusia, Protected Natural Area.
Esta aportación corológica se enmarca en un trabajo más amplio sobre la diversidad florística y fitosociológica de las comunidades vegetales del Espacio Natural Sierra de las Nieves, área que abarca el Parque Natural Sierra de las Nieves y la propuesta de Parque Nacional Sierra de las Nieves, en la provincia de Málaga (España) (Fig. 1). La mayoría de las novedades que comentamos son fruto de las recolecciones efectuadas entre los años 2017-2019, así como de la revisión de material recolectado anteriormente y depositado en el Herbario de la Universidad de Málaga (MGC).

Para la identificación, nomenclatura, distribución y hábitats principales de los taxones tratados se ha seguido a Flora Iberica (Castroviejo, 19862019) y Flora de Andalucía Oriental (Blanca et al., 2011), para verificar las novedades corológicas se ha usado ANTHOS (www.anthos.es) para la bibliografía y GBIF (www.gbif.org) para la búsqueda de pliegos de herbario. Las novedades corológicas pueden ser a nivel autonómico (Andalucía), provincial (Málaga), área natural (cf. Blanca et al., 2011), fitogeográfico (cf. Pérez Latorre et al., 2019) o nuevas poblaciones de taxones raros o protegidos.

Se indican un total de 21 taxones, de los cuales uno es novedad para el sur de la Península Ibérica, siete son novedad para la provincia de Málaga y para uno se confirma su presencia en la provincia de Málaga. Es remarcable que para seis de estos taxones estas nuevas poblaciones representan su distribución más suroccidental en la Península Ibérica, con una marcada disyunción con el resto de poblaciones peninsulares, de estas, la mitad tienen su óptimo en comunidades orófilas y/o higrófilas. Para cada taxón se indica

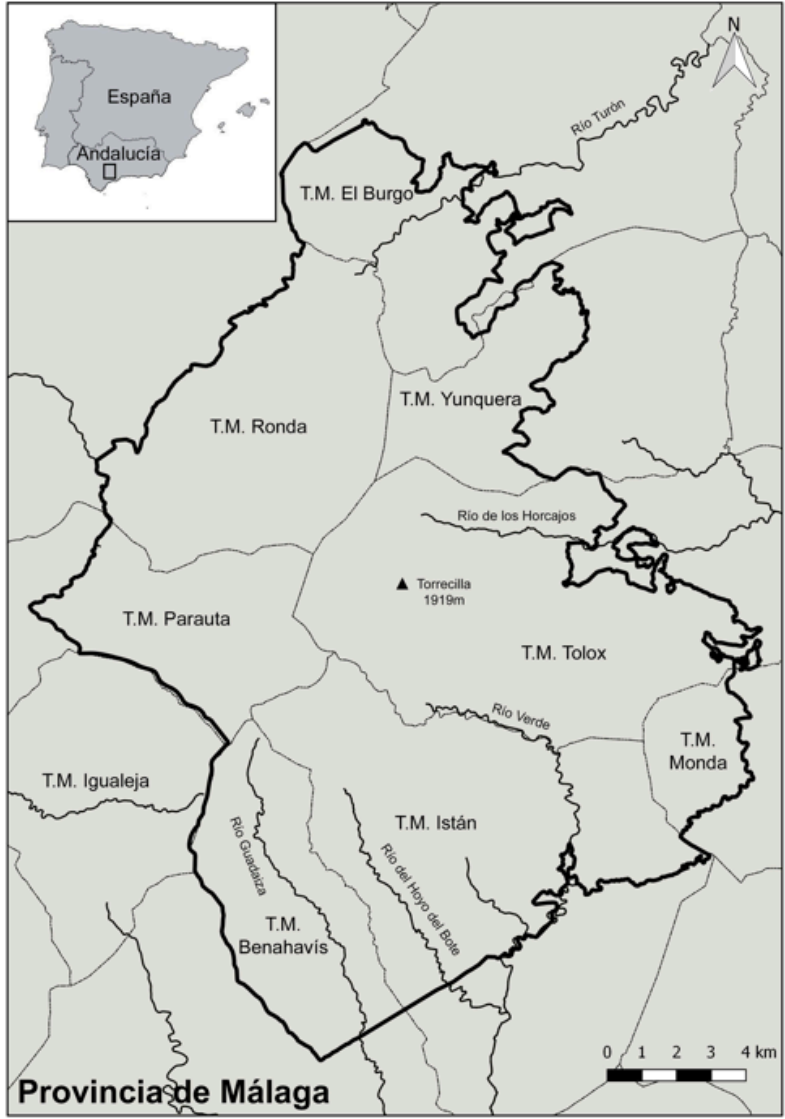

Figura 1. Límites del Espacio Natural Sierra de las Nieves (extraido de Guerra et al., 2018). T.M. (Término municipal).

Figure 1. Limits of the Natural Area Sierra de las Nieves (from Guerra et al., 2018), T.M. (Municipal district). 


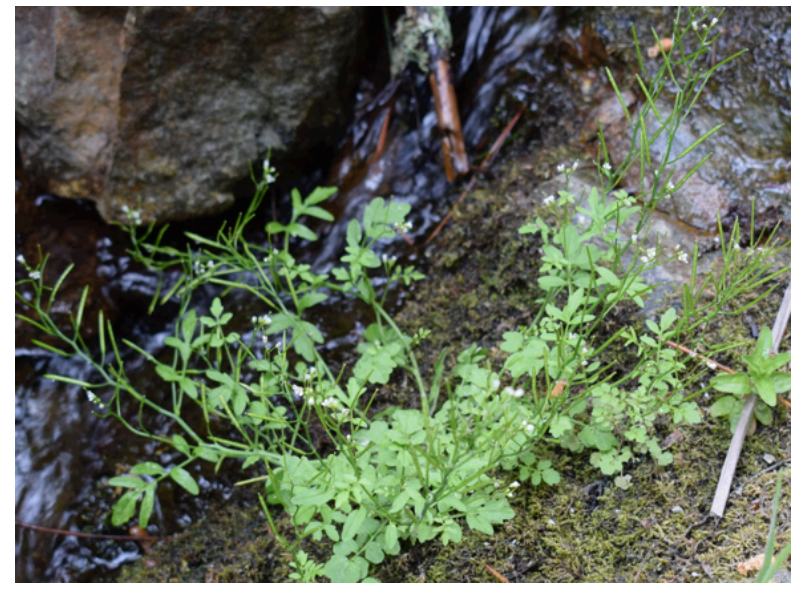

Figura 2. Cardamine flexuosa With. (Arroyos del Realejo)

su tipo biológico, distribución general, hábitat típico (fitosociológico) donde se ha localizado, un comentario corológico y/o taxonómico, y en su caso categoría de protección (Decreto 23/2012,14 febrero, Junta de Andalucía).

\section{Cardamine flexuosa With. (Fig. 2).}

Helófito erecto. Euroasiática y Alto Atlas marroquí. Vegetación tapizante de arroyos de montaña. Especie que en la mitad sur de la Península Ibérica solo se encuentra en Sierra Nevada (Granada) (Rico, 1993) y citada por Willkomm \& Lange (1861-1880, III: 825, sub. C. sylvatica Link.) en la Serranía de Ronda, dentro de los límites del actual Parque Natural: Sierra de Yunquera, en el Pilar de Tolox, localidad situada a $1700 \mathrm{~m}$, en el piso oromediterráneo con suelos arcillosos. Posteriormente no había vuelto a ser localizada (Rico, 1993; Pérez Latorre et al., 1998; Morales Torres, 2011a). La nueva localidad está situada en el piso meso-supramediterraneo a unos $1300 \mathrm{~m}$ sobre gneises, en arroyos con agua permanente ocupados por una comunidad dominada por Athyrium filix-femina (L.) Roth (Montio-Cardaminetea Br.-BI. \& Tüxen 1948).

Málaga: Igualeja, cara norte del Peñón del Robledal, La Fuenfría, arroyos en el margen derecho del arroyo del Realejo, 30SUF1656, 1200-1300 m, comunidades higrófilas, arroyo con agua permanente, suelos profundos, gneises, 09/06/2017, F. Soriguer, A. Picornell \& A. Garrido (MGC 86532; MGC 86536); Ibidem, 29/06/2017, B. Cabezudo \& F. Soriguer (MGC 87039)

\section{Centranthus nevadensis Boiss. subsp. nevadensis}

Caméfito sufruticoso. Sur de la Península Ibérica (Andalucía) y Noroeste de África. Rupícola en los pisos supra y oromediterráneo. Presente en las provincias de Almería, Cádiz, Granada y Málaga (López Martínez \& Devesa, 2007). En la Serranía de Ronda se encuentran sus poblaciones más occidentales, confinadas a las zonas más elevadas de la Sierra de Grazalema y Sierra de las Nieves, donde es muy escasa y localizada (Aparicio, 1993; Pérez Latorre et al., 1998). En la Sierra de las Nieves sensu stricto solo conocíamos un núcleo poblacional en el Picacho de Fatalandar (Pérez Latorre et al., 1998), la nueva población es más numerosa y se localiza en el Cerro de Estepilar y con la misma ecología, paredones calizos verticales con exposición norte (Rhamno pumilae-Saxifragetum granatesis Pérez Latorre \& Cabezudo 1998).

Málaga: Tolox, Parque Natural Sierra de las Nieves, Cerro del Oso (Estepilar), 30S UF2262, $1600 \mathrm{~m}$, tajos verticales y extraplomados, rupícola, 02/07/2014, B. Cabezudo, A. V. Pérez Latorre \& F. Soriguer (MGC 79632).

\section{Crypsis schoenoides (L.) Lam.}

Terófito cespitoso. Sur de Europa, $\mathrm{N}$ de África y Asia. Pastizales sobre suelos temporalmente inundados. Muy rara en la provincia de Málaga (Cabezudo et al., 2010), y no citada en la comarca de Ronda (Romero, 2011). La hemos recolectado en comunidades higronitrófilas en suelos con encharcamiento temporal (Trifolio fragiferi-Cynodontion Br.-Bl- \& O. Bólos 1958), con esta cita se amplían sus localidades en la provincia, siendo novedad para el área natural de Ronda.

Málaga: El Burgo, P. N. Sierra de las Nieves, Río Turón, azud del aprisco de la Hierbabuena, 30SUF2203571636, $636 \mathrm{~m}$, cola del azud, pastizales sobre suelos encharcados, limos, comunidades higro-nitrófilas, 13/07/2017, $F$. Soriguer \& M. Pulgar (MGC 87217); Ibidem, azud a los pies del mirador del Guarda Forestal, 30SUF2296672123, 13/07/2017, F. Soriguer \& M. Pulgar (MGC 87222).

\section{Epilobium lanceolatum Sebast. \& Mauri}

Hemicriptófito. Sur y Oeste de Europa, Norte de África, Caucaso y Anatolia. Especie muy localizada en el sur de la Península Ibérica, donde Nieto Feliner (1997) solo indicaba su presencia en Sierra Nevada (Granada), existiendo una cita sin pliego de referencia, del pinsapar de Grazalema en Cadiz (Rigueiro, 1978), cita que ha sido recientemente confirmada (Sánchez \& Fernández, 2016). Romero (2009) la cita también de la Sierra de las Nieves (Málaga) en base a un solo pliego de herbario (MGC) recolectado en 2002. Este año se ha vuelto a localizar en dos localidades en el piso 
oromediterráneo, una en la Sierra de las Nieves en el seno de sabinares postrados con pinsapos ( Abieto-Juniperetum sabinae Pérez Latorre \& Cabezudo 1998) y otra en la Sierra de Tolox sobre gleras (Thlaspietalia rotundifolii Br.-BI. 1936).

Málaga: Tolox, P.N. Sierra de las Nieves, camino del Pilar de Tolox al Picacho, 30SUF2261, $1750 \mathrm{~m}$. 2/07/2002, P. Navas, D. Navas, B. Cabezudo \& A. V. Pérez Latorre (MGC 52287); Ronda, P.N. Sierra de las Nieves, entre las cabeceras de la Cañada de Enmedio y de la Ánimas, 30S UF1962, 1735-1780 m. 5/06/2019, F. Soriguer \& A. V. Pérez Latorre (MGC 89598); Tolox, P.N. Sierra de las Nieves, cara norte del Torrecilla, 30SUF2161, 1780-1810 m., sabinares postrados y gleras,19/06/2019, $B$. Cabezudo, F. Soriguer, N. Hidalgo \& A. V. Pérez Latorre (MGC 90145) ; Ibidem, desde el Pilar de Tolox al Pto. de los Valientes, 1700-1750 m. (MGC 90146)

\section{Galega africana Mill.}

Hemicriptófito escaposo. Ibero-magrebí. Herbazales de quejigares y alcornocales. En la Península lbérica, se conoce de la provincia de Cádiz, desde el Puerto de Santa María a Algeciras (García \& Talavera, 1999) y de la provincia de Málaga, rara y muy localizada en el Valle del Genal (Gavira \& Pérez Latorre, 2003). Al igual que en las poblaciones del Valle del Genal, la nueva población aparece asociada a comunidades arbustivas higrófilas (Salicion pedicellatae Galán et al., 1999), hábitat desviante con respecto a las poblaciones gaditanas, con esta nueva localidad se amplía la distribución peninsular de esta especie, siendo esta nueva población la más oriental de la Península.

Málaga: Benahavís, Río Guadaiza, cola del pantano, 30SUF2145, saucedas y tarajales, gravas y arenas, 9/06/2014, B. Cabezudo \& F. Soriguer (MGC 79464).

\section{Helianthemun papillare Boiss.}

Terófito fasciculado. Ibero-Magrebí. Asociado a pastizales secos sobre calizas, dolomías o yesos, en zonas generalmente montanas. En la Península Ibérica, circunscrita al sureste, donde no es rara, estando citada en Andalucía en las provincias de Almería, Granada y Jaén (López González, 1993; Morales Torres, 2011b). Lo hemos recolectado en una única localidad en la Sierra de las Nieves sensu stricto, en su hábitat típico de pastizales terofíticos oligótrofos (Trachynion distachyae Rivas-Mart.1978). Cita nueva para la provincia de Málaga y la localidad más occidental de la especie en la Península Ibérica.

Málaga: Yunquera, P. N. Sierra de las Nieves, Sierra del Pinar, camino del puerto del Saucillo a Enamorados, entre el puerto y el límite del pinsapar, 30SUF2361965242, $1520 \mathrm{~m}$, pastizales anuales, 31/05/2017, B. Cabezudo \& F. Soriguer (MGC 86238).

\section{Heliotropium supinum $\mathrm{L}$.}

Terófito reptante. Distribuido ampliamente por el Mediterráneo. Pastizales sobre suelos temporalmente inundados. En la provincia de Málaga estaba citado, en las vegas de los ríos Guadalhorce y Vélez por Willkomm \& Lange (1861-1880, II: 513), y, sin confirmar, en el área natural de Ronda (Valdés, 2011), presencia que confirmamos con esta cita. La hemos recolectado en comunidades higronitrófilas en suelos con encharcamiento temporal (Trifolio fragiferiCynodontion).

Málaga: El Burgo, P. N. Sierra de las Nieves, río Turón, azud a los pies del mirador del Guarda Forestal, 30SUF2296672123, $636 \mathrm{~m}$, cola del azud, suelos limosos encharcados, 20/06/2017, B. Cabezudo \& F. Soriguer (MGC 86754); Ibidem, 13/07/2017, F. Soriguer \& M. Pulgar (MGC 87223); lbidem, azud del aprisco de la Hierbabuena, 30SUF2203571636, 610 m, cola del azud, pastizales sobre suelos encharcados, limos, comunidades higronitrófilas, 20/06/2017, B. Cabezudo \& F. Soriguer (MGC 86811); Ibidem, 13/07/2017, F. Soriguer \& M. Pulgar (MGC 87220)

\section{Juncus rechingeri Snogerup}

Terófito cespitoso. Asia central, Oriente próximo, Marruecos y Península Ibérica. Pastizales húmedos en zonas con encharcamiento temporal. Pertenece al complejo de J. bufonius L., siendo citado para Europa recientemente (Romero Zarco, 2008). El principal carácter diferencial con las otras especies del grupo es la marcada ornamentación estriado-reticulada de las semillas maduras (cf. Romero Zarco, 2008, 2010), carácter que nos ha permitido adjudicar claramente nuestro material a este taxón. En Andalucía está presente en las provincias de Almería, Granada y Jaén (Romero Zarco, 2010). La hemos recolectado en una única localidad en la Sierra de las Nieves sensu stricto, en su hábitat típico de suelos húmedos (IsoetoNanojuncetea Br.-BI. \& Tüxen 1946). Se trata de una cita nueva para la provincia de Málaga y la localidad más suroccidental de la especie en la Península lbérica.

Málaga: Yunquera, P. N. Sierra de las Nieves, vereda del Cortijo de Huarte al Peñon de Ronda, Las Arenitas, 30SUF2208066916 (rad. pto.: 650 $\mathrm{m})$, $950-1150 \mathrm{~m}$, suelos arenosos húmedos, dolomías, 22/6/2018, B. Cabezudo \& F. Soriguer (MGC 88953) 
Lamium bifidum Cirillo (Fig. 3).

Terófito. Centro y este de la región mediterránea y Península Ibérica, donde se circunscribe al centro y el oeste. Herbazales escionitrófilos en ambientes boscosos. No citado con anterioridad en el sur de la Península Ibérica (Ubera, 1987; Pujadas, 2010; Navarro, 2011a; Cueto et al., 2018). Lo hemos recolectado en su hábitat típico, sotobosque de quejigales y alcornocales (Quercion suberis Loisel 1971), siendo esta cita novedad para Andalucía y la localidad más al sur de la Península Ibérica, donde representa una notable disyunción.

Málaga: Istán. Vertiente norte del Cerro del Duque, alcornocal-quejigal del arroyo de la Cruz, frente a la Majada del Algarrobo, orientación norte, 30 S UF 1957, $760 \mathrm{~m}$, alcornocal-quejigal y escobonales, gneises, 29/03/2019, B. Cabezudo, F. Soriguer \& M. Recio (MGC 89329); Ibidem, 10/04/2019, B. Cabezudo, F. Soriguer \& A. V. Pérez Latorre (MGC 89330).

\section{Lepidium calycotrichum Kunze subsp. anticarium} Valdés Berm. \& G. Lopez

Hemicriptófito escaposo. Endemismo del sur de la Península Ibérica, provincia de Málaga, subsector torcalense (cf. Pérez Latorre et al., 2019). Vegetación de roquedos calizos. Hasta ahora solo se conocía de la Cordillera Antequerana, desde la sierra del Jobo hasta la Sierra de Huma (Hernandez Bermejo \& Clemente, 1993; Pérez Latorre et al., 2014). Lo hemos recolectado en el seno de comunidades umbrófilas subrupícolas (Campanulion velutinae MartínezParras \& Peinado 1990). Con la nueva localidad que citamos, se amplía su presencia a la Serranía de Ronda y al sector Rondeño, siendo esta su localidad más occidental cerca del contacto con el sector Antequerano. En el resto de la zona estudiada todo el material revisado hasta la fecha corresponde a la subespecie nominal.

Málaga: Ronda, P. N. Sierra de las Nieves, Valle de Lifa, Sierra Hidalga, desde el Puerto de Lifa al Carramolo del Queso, 30SUF1704768581(rad. pto.: $350 \mathrm{~m}$ ), 1160-1324 m, lastonares sobre lapiaces, calizas, 24/5/2018, F. Soriguer \& $M$. Pulgar (MGC 88364).

\section{Marrubium supinum $\mathrm{L}$.}

Caméfito sufruticoso. Mediterráneo occidental. Matorrales y pastizales nitrificados en zonas montanas. En la Península Ibérica se distribuye por el centro y este, en Andalucía se presenta en su mitad oriental, circunscrito a sus zonas montañosas (Navarro, 2011b; Morales, 2010). Sus poblaciones más occidentales conocidas hasta ahora se encontraban en el sector Torcalense, en

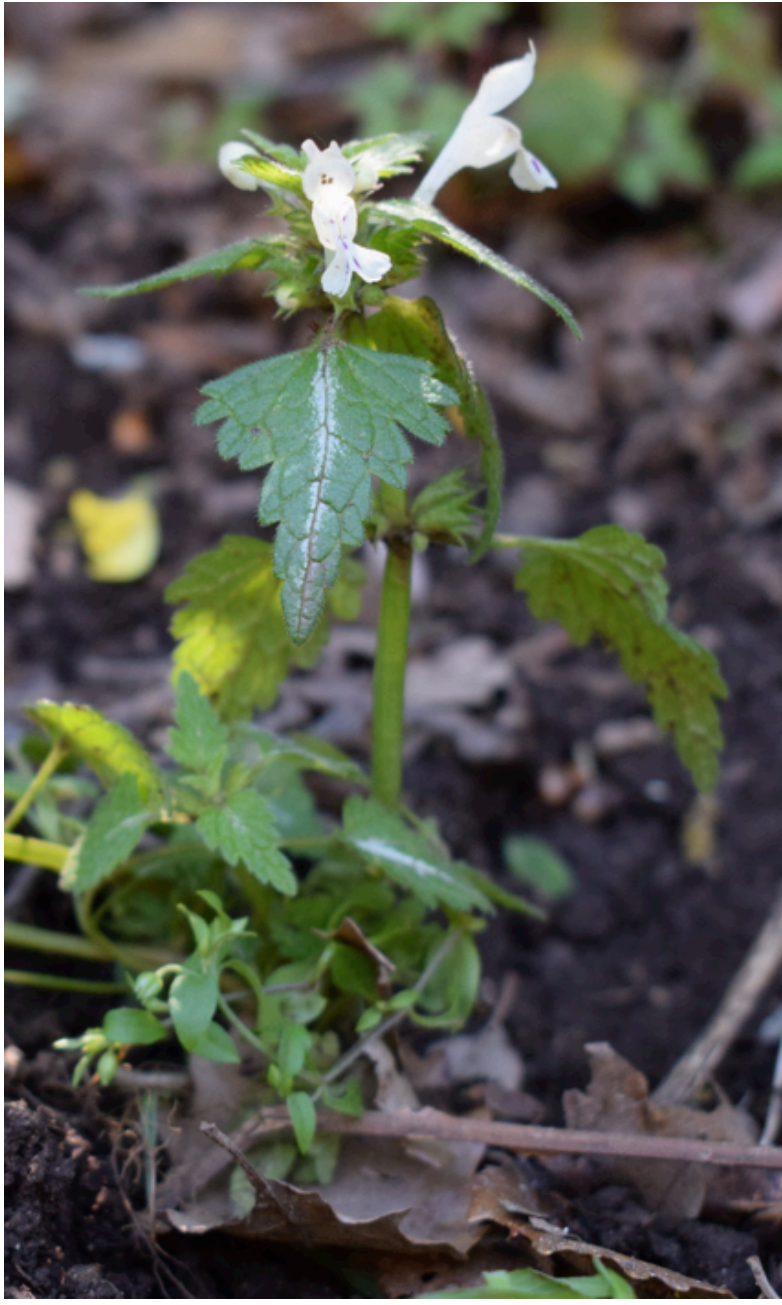

Figura 3. Lamium bifidum Cirillo (Majada del Algarrobo)

la Sierra del Valle de Abdalajís (Cabezudo et al., 2011). Hasta ahora no había referencias sobre su presencia en la Serranía de Ronda, con esta nueva localidad se amplía su presencia hasta el sector Rondeño, y en su hábitat típico, matorrales muy pastoreados (Helichryso stoechadis-Santolinetalia squarrosae Peinado \& Martínez-Parras 1984).

Málaga: Ronda, P. N. Sierra de las Nieves, desde la cabecera de la Cañada de las Ánimas al Peñón de los Enamorados por el cresterío, 30SUF2040263527 (rad. pto.: 670 m) 1750-1813 $\mathrm{m}$, zona rocosa, gleras, roquedos, matorral-pastizal pastoreado, calizas, 19/5/2017, B. Cabezudo, F. Soriguer \& A. V. Pérez Latorre (MGC 85744).

Milium vernale $M$. Bieb. subsp. montianum (Parl.) K. Richt.

Terófito. Mediterránea. Repartida principalmente por el centro y norte de la Península Ibérica, estando restringida en Andalucía a algunas serranías de Andalucía oriental. En Málaga solo estaba citada en la sierra de Camarolos (Cabezudo 
et al., 2016). Para el sector Rondeño no teníamos referencias previas. La hemos recolectado en una sola localidad y muy escasa, en pastizales supramediterraneos (Poetalia bulbosae Rivas Goday \& Rivas Martinez 1970).

Málaga: El Burgo, Sierra Blanquilla, desde el Cortijo de Bolina al Cerro del Viento, 20SUF1872, 1240-1427 m., pequeñas dolinas, pastizales, suelos arcillosos, calizas, 29/5/2019, B. Cabezudo, F. Soriguer, \& A. V. Pérez Latorre (MGC 90147).

\section{Orchis cazorlensis Lacaita}

Geofito. Endemismo ibérico, distribuido por algunas serranías de la mitad este y sur de la Península Ibérica. En Andalucía se localiza fundamentalmente en las serranías orientales, con una localidad disyunta en la Sierra de las Nieves (Algarra \& Blanca, 2011), con una sola población conocida, asociada a matorrales abiertos en el piso mesomediterraneo (Borrego et al., 2007). Hemos localizado otra población en sabinares postrados oromediterraneos con pinsapos (AbietoJuniperetum sabinae)

Málaga: Tolox, P.N. Sierra de las Nieves, Sierra de Tolox, vertiente sur del Torrecilla, vereda de Pto. Corona al Pto. de los Valientes, 30SUF2261, 16501700 m., sabinar postrado con pinsapos, calizas, 14/06/2019, B. Cabezudo \& F. Soriguer (MGC 90149)

\section{Ornithogalum reverchonii Lange ex Willk.}

Geófito bulboso. Bético-Magrebí. Roquedos calcáreos en grietas y repisas terrosas. En la Peninsula Íberica se presenta en la Serranía de Ronda, y en una localidad disyunta en la Sierra de Cazorla (Martínez Azorín et al., 2007, 2013). En la Serranía de Ronda todas las citas confirmadas que conocemos se concentran en su parte occidental, desde la Sierra de Grazalema a los alrededores de Ronda y cuenca alta del río Genal (Gavira \& Pérez Latorre, 2003; Martínez Azorín et al., 2007; Becerra \& Robles, 2007). En el ámbito del Parque Natural Sierra de las Nieves no se conocía su presencia (Pérez Latorre et al., 1998), aunque existe una referencia bibliográfica reiterada (Moreno \& Soriano, 1989; Martínez Azorín et al., 2007): Sierras de Tolox y Yunquera, sin más datos, proveniente de Asensi (1976), sin pliego de referencia. La población encontrada ocupa repisas terrosas en grandes cortados rocosos con orientación norte (Saxifragion camposii Cuatrecasas 1953). Esta nueva población pasa por ser la única conocida en el ámbito del Parque Natural y la más oriental de la Serranía de Ronda. Especie Vulnerable (legislación andaluza)

Málaga: Ronda, P. N. Sierra de las Nieves, cara norte y oeste del Peñón de Ronda y alrededores del Cortijo del Peñón de Ronda, 30 SUF2114367030 (rad. pto:: $400 \mathrm{~m}$ ), comunidades rupícolas en pequeñas repisas, calizas, 16/5/2018, $B$. Cabezudo, F. Soriguer \& A. V. Pérez Latorre (MGC 88087).

Platycapnos tenuiloba L. subsp. paralella Lidén Terófito erecto. Endemismo rondeño y almijarogranatense. Pastizales generalmente sobre arenas dolomíticas. Presente solo en la Serranía de Ronda (Sierra de Alcaparaín), Sierra de Mijas y Sierra de Almijara, provincias de Málaga y Granada (Salinas, 2011). No se conocía su presencia en el ámbito del Parque Natural Sierra de las Nieves (Pérez Latorre et al., 1998) aunque sí en sus proximidades (Venta Añoreta), localidad citada en la descripción del taxón (Lidén, 1984), donde se indica además la peculiaridad del substrato donde habitaba: harder and less crystalline limestone (calizas duras $y$ menos cristalinas). Hemos hallado 2 núcleos poblacionales dentro del Parque Natural Sierra de las Nieves, y en el mismo hábitat (Omphalodion commutate Rivas-Mart. et al. 1987) que indicaba Lidén (1984). Especie en Régimen de Protección Especial (legislación andaluza)

Málaga: Ronda, P. N. Sierra de las Nieves, Sierra de la Hidalga, subida desde el repetidor del Puerto del Pilón a la cumbre de la Hidalga, 30SUF1449565681, 1350-1400 m, roquedos y gleras, dolomías, 16/5/2017, B. Cabezudo, J. García-Sánchez \& F. Soriguer (MGC 85600); El Burgo: Sierra Blanquilla, carril de los Llanos de Manuel Mingo, fin del carril, 30SUF1972, 1200 m, roquedos, calizas, 10/5/2018, B. Cabezudo \& F. Soriguer (MGC 87848).

\section{Ribes uva-crispa L. subsp. austro-europaeum (Bornm.) Bech.}

Nanofanerófito caducifolio. Europa, Asia Central y China, Península Ibérica y la alta montaña marroquí. Espinares caducifolios y bosques caducifolios climatófilos, en los pisos supra y oromediterráneo. En Andalucía se presenta solo en su mitad oriental, muy escasa y localizada en la alta montaña de las provincias de Almería, Granada y Jaén (Blanca, 1997, 2011). La hemos recolectado en una única localidad de la Sierra de las Nieves sensu stricto en su hábitat típico de espinares caducifolios de montaña (Pruno mahalebo-Berberidetum hispanicae Asensi \& RivasMart.1979). Muy escasa, la hemos recolectado sin fructificar, por lo que no podemos adjudicarlo a ninguna de las variedades reconocidas. Novedad para la provincia de Málaga y la localidad más suroccidental de la especie en la Península Ibérica. 
Málaga: Tolox, P. N. Sierra de las Nieves, Pilar de Tolox y arroyo de los Pilones, 30SUF2161, 1640-1695 m, espinares caducifolios, calizasarcillas, 16/6/2017, B. Cabezudo, F. Soriguer \& A. V. Pérez Latorre (MGC 86710).

Senecio lopezii Boiss. (Fig. 4)

Hemicriptófito escaposo. Endemismo del SW de la Península lbérica, presente en el Algarve portugués y provincias de Cádiz y Málaga en España. Pastizales esciófilos en ambientes nemorales. Sus poblaciones clásicas se concentran en el sector Aljíbico de la provincia de Cádiz, siendo más raro en la parte malagueña, y más escaso aun en el sector rondeño. Este Senecio fue descrito con material de la Sierra de las Nieves sensu stricto: Cerca de Yunquera y del Desierto de las Nieves (Boissier 1838; 1839-1845, II: 335), donde no había vuelto a ser localizado. Se ha vuelto a encontrar en el seno de comunidades umbrófilas sobre suelos descarbonatados ligadas a pinsapares (Paeonio broteroi-Abietetum pinsapo Asensi \& Rivas-Mart. 1976). Con esta cita se vuelve a confirmar la presencia de la especie en la zona, siendo la segunda población hasta el momento en el sector rondeño e igual de escasa (Cabezudo et al., 2010).

Málaga: Parauta, P. N. Sierra de las Nieves, alrededores del Pinsapo de las Escaleretas, 30SUF1859, $1150 \mathrm{~m}$, pinsapar y matorral, suelos descarbonatados, calizas, 1/6/2016, B. Cabezudo \& F. Soriguer (MGC 82990)

\section{Trifolium isthmocarpum Brot.}

Terófito escaposo. Península Ibérica, Italia, NW de África, Turquía y Madeira. Pastizales higrófilos. En la Península Ibérica ocupa el SW y en Andalucía está presente en las provincias occidentales de Cádiz, Huelva y Sevilla (Muñoz et al., 2000) y Jaén, en Sierra Morena (Muñoz, 2011). Esta nueva localidad amplía su distribución hasta la provincia de Málaga, donde es novedad. (Molinio-Holoschoenion Br.-BI. \& Tchou 1947).

Málaga: Istán, Monte de Bornoque, 30S UF25, 24/05/2000, Y. Gil, P. Navas \& D. Navas (MGC 50385).

\section{Trifolium ornithopodioides $\mathrm{L}$.}

Terófito. Eurosiberiana y Mediterránea. En la Península Ibérica se localiza principalmente en el centro y oeste. En Andalucía se localiza en el Bajo Guadalquivir (Huelva y Sevilla) donde al parecer es raro (Muñoz et al., 2000; Ruiz de Clavijo, 1987) y en la serranía de Cazorla, donde es muy raro (Muñoz, 2011). Hemos localizado una pequeña población asociada a pastizales sobre suelos

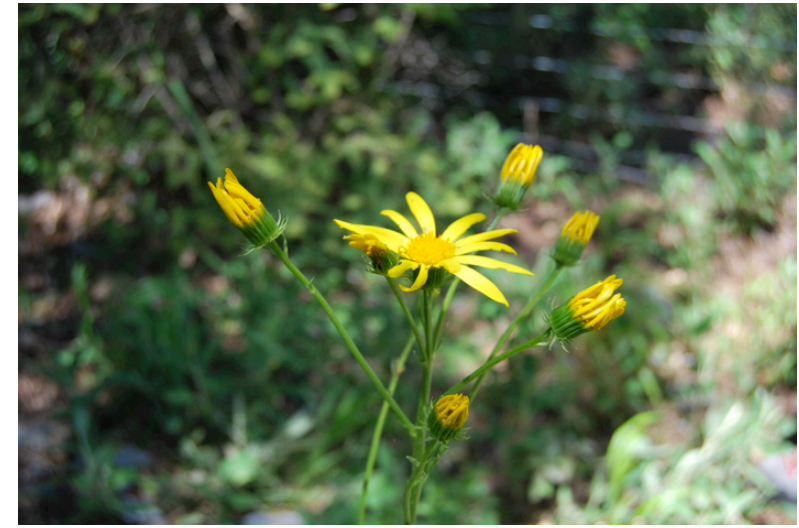

Figura 4. Senecio lopezii Boiss. (P. Natural Sierra de las Nieves)

arcillosos con encharcamiento temporal (IsoetoNanojuncetea) del piso oromediterráneo. Novedad para la provincia de Málaga. El hábitat donde la hemos recolectado es similar al de las poblaciones de Cazorla y desviante al de las poblaciones de Andalucía occidental.

Málaga: Tolox, P.N. Sierra de las Nieves, Quejigal de Tolox, vereda del Pto. de Pilones al Peñón de Enamorados 30SUF2063, 5/06/2019, F. Soriguer, \& A. V. Pérez Latorre (MGC 90148).

\section{Veronica sibthorpioides Debeaux, Degen \& Hervier}

Terófito reptante. Ibero-magrebí, centro-este y sureste de la Península Ibérica y Atlas marroquí. Pedregales en zonas montanas, generalmente calizos y pastoreados. En Andalucía presente en las provincias de Almería, Granada y Jaén (Martínez Ortega et al., 2009; Sánchez \& Martínez Ortega, 2011). Esta cita es novedad para Málaga y la localización peninsular más suroccidental de la especie. Esta zona, sometida a pastoreo, está ocupada por un mosaico de formaciones arbustivas orófilas y pastizales (Geranio purpureiCardaminetalia hirsutae Brullo 1985).

Málaga: Tolox, P. N. Sierra de las Nieves, vereda al Torrecilla, cercanías al Pilar de Tolox, 30SUF216, $1700 \mathrm{~m}$, calizas, 19/06/1997, A. V. Pérez Latorre, P. Navas, Y. Gil \& D. Navas (MGC 46199).

\section{Veronica verna $\mathrm{L}$.}

Terófito erecto. Euroasiática. Pastizales anuales generalmente silicícolas. En Andalucía solo citada en su parte más oriental (Martínez Ortega et al., 2009; Sánchez \& Martínez Ortega, 2011). Esta nueva localidad es novedad para la provincia de Málaga y pasa por ser la localidad más suroccidental de la Península Ibérica. El biotopo está sometido a pastoreo y está ocupado 
por un mosaico de formaciones arbustivas orófilas y pastizales (Tuberarietea guttatae Br.-BI. 1952).

Málaga: P. N. Sierra de las Nieves, Puerto de los Pilones, ladera Noroeste cerca del pico, sustrato calizo, $1720 \mathrm{~m}, 30$ SUF1962, 07/07/1997, A. V. Pérez Latorre, P. Navas, Y. Gil \& D. Navas (MGC 46126).

\section{Agradecimientos}

Agradecemos al director del P. Natural Sierra de las Nieves y a los agentes de Medio Ambiente, por facilitarnos el acceso a la zona estudiada y a J. García Sánchez y M. Pulgar por su ayuda en las labores de campo. Este trabajo se enmarca en un convenio Junta de Andalucía- Grupo de Investigación RNM-115 de la Universidad de Málaga para el estudio de la Flora y Vegetación del Espacio Natural Sierra de las Nieves.

\section{Bibliografía.}

ANTHOS (2019). Sistema de Información sobre las Plantas de España, http://www.anthos.es (último acceso abril 2019).

Algarra, J. \& Blanca, G. (2011). Orchis L. In G. Blanca, B. Cabezudo, M. Cueto, C. Morales Torres \& C. Salazar (eds.) Claves de la Flora Vascular de Andalucía Oriental. (pp. 90-92). Universidades de Granada, Almería, Jaén y Málaga. Granada.

Aparicio, A. (1993). Planes de recuperación de especies vegetales amenazadas en el Parque Natural de la Sierra de Grazalema (Cádiz-Málaga.) Acta Botanica Malacitana, 18, 199-221.

Asensi A. (1976). Flora y Vegetación de las áreas ocupadas por el Abies pinsapo Boiss. Tesis doctoral. Universidad de Granada.

Becerra Parra, M. \& Robles Domínguez, E. (2007). Catálogo florístico del Macizo de Líbar (Parques Naturales Sierra de Grazalema y Los Alcornocales, Málaga-Cádiz, España) Acta Botanica Malacitana, 32, 161-200.

Blanca, G. (1997). Ribes L. In Castroviejo (Coord.) Flora ibérica 5, 86-94. Real Jardín Botánico, CSIC, Madrid.

Blanca, G., Cabezudo, B., Cueto, M., Salazar, C. \& Morales Torres, C. Eds. (2011). Flora Vascular de Andalucía Oriental, $2^{\mathrm{a}}$ ed. Universidades de Almería, Granada, Jaén y Málaga, Granada, 1751 pp.

Blanca, G. (2011). Ribes L. In G. Blanca, B. Cabezudo, M. Cueto, C. Morales Torres \& C. Salazar (eds.) Claves de la Flora Vascular de Andalucía Oriental. (pp. 250-251). Universidades de Granada, Almería, Jaén y Málaga. Granada.

Boissier, P.E. (1838) Elenchus plantarum novarum. Genéve.

Boissier, P.E. (1839-1845) Voyage botanique dans le midi de l'Espagne pendant l'année 1837. 2 vol.. Paris.

Borrego Aguayo, F., López Pastora, A., Melgar Rueda, M., Prats Fons, S., Sierra de Cózar \& Terroba Valadés, J. (2007). Orchis cazorlensis Lacaita en el Parque Natural Sierra de las Nieves (Málaga). Acta Botanica
Malacitana 32, 267-268.

Cabezudo, B., Pérez Latorre, A.V., Casimiro-Soriguer Solanas, F., Gavira, O., García Rojas, J.A. \& Pavón, M. (2010). Nota sobre Abies pinsapo Clemente ex Boiss. y otras plantas andaluzas, Acta Botanica Malacitana, 35, 195-204.

Cabezudo, B., Pérez Latorre, A.V., Casimiro-Soriguer Solanas, F., Pavón, M. \& Hidalgo Triana, N. (2011). Algunas plantas interesantes de Andalucía Oriental (Málaga-Granada). Acta Botanica Malacitana, 36, 213-217.

Cabezudo, B., Casimiro-Soriguer Solanas, F., García Sanchez, J. \& Pérez Latorre A.V. (2016). Flora y Vegetación de la Zona de especial Conservación Sierra de Camarolos. Acta Botanica Malacitana, 41, 163-246.

Castroviejo, S. (Coord.) (1986-2019), Flora iberica, vols. 1-21. Real Jardín Botánico, CSIC, Madrid.

Cueto, M., Melendo, M., Giménez, E., Fuentes, J., López Carrique, E. \& Blanca, G. (2018) First updated checklist of the vascular flora of Andalusia (S of Spain). Phytotaxa, 339 (1), 01-95.

García Murillo, P. \&Talavera, S. (1999). Galega L. In S. Castroviejo (Coord.) Flora iberica 7 (1): 277-273. Real Jardín Botánico, CSIC, Madrid.

Gavira Romero, O. \& Pérez Latorre A.V. (2003). Aproximación al catálogo florístico del Valle del río Genal (Serranía de Ronda, Málaga, España). Anales de Biología, 25, 113-161.

GBIF (2019) The Global Biodiversity Information Facility, http://www.gbif.org (último acceso abril 2019).

Guerra, J., Gallego, M.T., Jiménez, J.A., Cano, M.J., Casimiro-Soriguer Solanas, F., Pérez Latorre, A.V. \& Cabezudo, B. (2018). Flora Briofítica del Espacio Natural Sierra de las Nieves y su entorno (Málaga, España). Universidad de Murcia. 27 pp.

Hernández Bermejo, J.E. \& Clemente, C. (1993) Lepidium L. In Castroviejo (Coord.). Flora iberica 4: 311-327. Real Jardín Botánico, CSIC, Madrid.

Lidén, M. (1984). Novelties on Fumariodeae, Anales del Jardín Botánico de Madrid, 41(1), 221-222.

López González, G. (1993). Helianthemun Mill. In Castroviejo (Coord.) Flora iberica 3: 365-421. Real Jardín Botánico, CSIC, Madrid.

Lopez Martínez, J \& Devesa J.A. (2007). Centranthus DC. In Castroviejo (Coord.) Flora iberica 15: 223-232. Real Jardín Botánico, CSIC, Madrid.

Martínez Azorín, M., Crespo, M.B. \& Juan, A. (2007). Taxonomic revision of Ornithogalum subg. cathissa (Salisb) Baker, Anales Jardín Botánico Madrid 64(1), 7-25

Martínez Azorín, M., Crespo, M.B. \& Juan, A. (2013). Ornithogalum L. In Castroviejo (Coord.) Flora iberica 20: 188-207. Real Jardín Botánico, CSIC, Madrid.

Martínez Ortega, M., Sánchez agudo, J.A. \& Rico, E. (2009) Veronica L. In Castroviejo (Coord.) Flora iberica 13: 360-432. Real Jardín Botánico, CSIC, Madrid.

Morales, R. (2010). Marrubium L. In Castroviejo (Coord.) Flora iberica 7 (2): 288-292. Real Jardín Botánico, CSIC, Madrid.

Morales Torres, C. (2011a). Cardamine L. In G. Blanca, B. Cabezudo, M. Cueto, C. Morales Torres \& C. Salazar 
(eds.) Claves de la Flora Vascular de Andalucía Oriental, pp. 363. Universidades de Granada, Almería, Jaén y Málaga. Granada.

Morales Torres, C. (2011). Helianthemun Mill. In G. Blanca, B. Cabezudo, M. Cueto, C. Morales Torres \& C. Salazar (Eds.) Claves de la Flora Vascular de Andalucía Oriental, pp. 397-403. Universidades de Granada, Almería, Jaén y Málaga. Granada.

Moreno Saiz, J.C. \& Soriano, C. (1989). Asientos para un Atlas Corológico de la Flora Occidental. Mapa 118, Fontqueria 22: 20-21.

Muñoz Rodríguez, A. (2011). Trifolium L. In G. Blanca, B. Cabezudo, M. Cueto, C. Morales Torres \& C. Salazar (Eds.) Claves de la Flora Vascular de Andalucía Oriental, (pp. 305-310). Universidades de Granada, Almería, Jaén y Málaga. Granada.

Muñoz Rodríguez, A., Devesa, J.A. \& Talavera, S. (2000). Trifolium L. In Castroviejo (Coord.) Flora iberica 7 (2): 647-719. Real Jardín Botánico, CSIC, Madrid.

Navarro, T. (2011a). Lamium L. Aiton. In G. Blanca, B. Cabezudo, M. Cueto, C. Morales Torres \& C. Salazar (Eds.) Claves de la Flora Vascular de Andalucía Oriental, (pp. 495-496). Universidades de Granada, Almería, Jaén y Málaga. Granada.

Navarro, T. (2011b). Marrubium L. In G. Blanca, B. Cabezudo, M. Cueto, C. Morales Torres \& C. Salazar (Eds.) Claves de la Flora Vascular de Andalucía Oriental, pp. 500. Universidades de Granada, Almería, Jaén y Málaga. Granada.

Nieto Feliner, G. (1997). Epilobium L. In S. Castroviejo (Coord.) Flora iberica 8: 101-131. Real Jardín Botánico, CSIC, Madrid.

Pérez Latorre, A.V., Navas, P., Navas, D., Gil, Y. \& Cabezudo, B. (1998). Datos sobre flora y vegetación de la Serranía de Ronda (Málaga, España). Acta Botanica Malacitana 23, 149-191.

Pérez Latorre, A.V., Casimiro-Soriguer Solanas, F., García Sánchez, J. \& Cabezudo, B. (2014). Flora y Vegetación del Paraje Natural Desfiladero de los Gaitanes y su entorno (Málaga, España). Acta Botanica Malacitana 39, 129-177.

Pérez Latorre, A.V., Hidalgo Triana, N. \& Cabezudo, B. (2019). Mapa biogeográfico de la provincia de Málaga. Universidad de Málaga y Diputación de Málaga.

Pujadas A.J. (2010). Lamium L. In Castroviejo (Coord.) Flora iberica 12, 181-196. Real Jardín Botánico, CSIC, Madrid.

Rico, E. (1993). Cardamine L. In Castroviejo (Coord.) Flora iberica 4, 119-131. Real Jardín Botánico, CSIC,
Madrid.

Rigueiro Rodriguez, A. (1978). Catalogo floristico de la Sierra de Grazalema, Trab. Catedra Bot. Esc. Tecn. Super. Ing. Montes 4, 1-272.

Romero, A.T., (2009). Epilobium L. In G. Blanca, B. Cabezudo, M. Cueto, C. Morales Torres \& C. Salazar (Eds.) Flora Vascular de Andalucía Oriental, (pp. 243-247). Consejería de Medio Ambiente, Junta de Andalucía, Sevilla.

Romero, A.T., (2011). Crypsis Aiton In G. Blanca, B. Cabezudo, M. Cueto, C. Morales Torres \& C. Salazar (Eds.) Claves de la Flora Vascular de Andalucía Oriental, pp. 161. Universidades de Granada, Almería, Jaén y Málaga. Granada.

Romero Zarco, C. (2008). Juncus rechingeri Snogerup, nueva especie para la Flora Europea, Acta Botanica Malacitana, 33, 339-341.

Romero Zarco, C. (2010). Juncus L. In Castroviejo (Coord.) Flora ibérica 17, 123-186. Real Jardín Botánico, CSIC, Madrid.

Ruiz de Clavijo, E. (1987) Trifolium L. In: Valdés, B. Talavera, S. \& Galiano, E.F. (Eds.) Flora Vascular de Andalucía Occidental, 2 (pp.508-522). Ketres, Barcelona,

Salinas, M.J. (2011). Fumariaceae In G. Blanca, B. Cabezudo, M. Cueto, C. Morales Torres \& C. Salazar (Eds.) Claves de la Flora Vascular de Andalucía Oriental (pp. 185-189). Universidades de Granada, Almería, Jaén y Málaga. Granada.

Sanchez Agudo, J.A. \& Martinez-Ortega, M. (2011). Veronica L. In G. Blanca, B. Cabezudo, M. Cueto, C. Morales Torres \& C. Salazar (Eds.) Claves de la Flora Vascular de Andalucía Oriental. (pp. 467-471). Universidades de Granada, Almería, Jaén y Málaga. Granada.

Sanchez García, I. \& Fernández de Bobadilla (2016). Tres especies nuevas para la Flora de Andalucía Occidental. Revista de la Sociedad Gaditana de Historia natural, 10, 65-69.

Ubera, J. (1987). Lamium L. In Valdés, B., Talavera, S. \& Galiano, E.F. (Eds.) Flora Vascular de Andalucía Occidental. vol.2. (pp.508-522). Ketres, Barcelona.

Valdés, B. (2011). Boraginaceae In G. Blanca, B. Cabezudo, M. Cueto, C. Morales Torres \& C. Salazar (Eds.) Claves de la Flora Vascular de Andalucía Oriental, pp. 443-453. Universidades de Granada, Almería, Jaén y Málaga. Granada.

Willkomm, M. \& Lange, J. (1861-1880). Prodromus Florae Hispanicae. 3 vols. Stuttgart, Alemania. 